
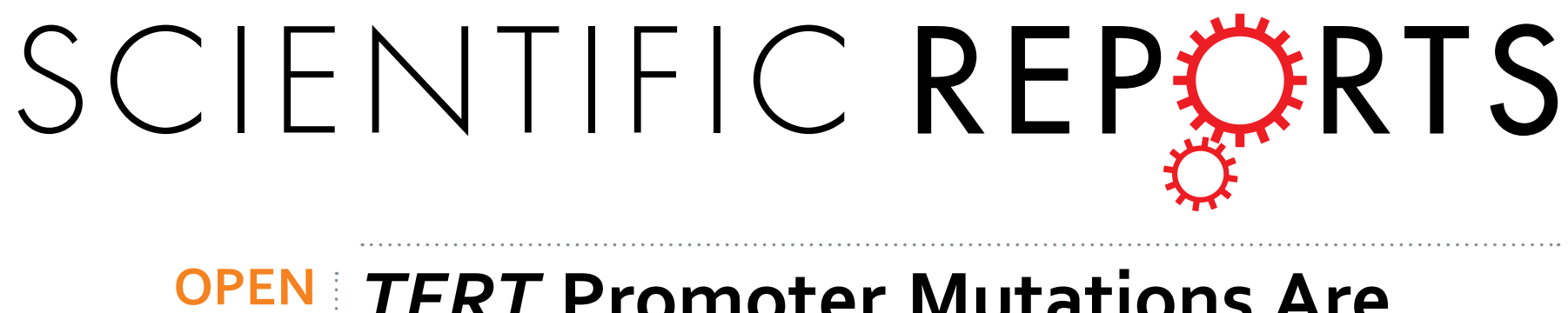

\title{
TERT Promoter Mutations Are Predictive of Aggressive Clinical Behavior in Patients with Spitzoid Melanocytic Neoplasms
}

Received: 29 January 2015

Accepted: 30 April 2015

Published: 10 June 2015

\begin{abstract}
Seungjae Lee ${ }^{1}$, Raymond L. Barnhill ${ }^{2}$, Reinhard Dummer ${ }^{3}$, James Dalton ${ }^{1}$, Jianrong $\mathrm{Wu}^{4}$, Alberto Pappo 5 \& Armita Bahrami ${ }^{1}$
\end{abstract}

Spitzoid neoplasms constitute a morphologically distinct category of melanocytic tumors, encompassing Spitz nevus (benign), atypical Spitz tumor (intermediate malignant potential), and spitzoid melanoma (fully malignant). Currently, no reliable histopathological criteria or molecular marker is known to distinguish borderline from overtly malignant neoplasms. Because TERT promoter (TERT-p) mutations are common in inherently aggressive cutaneous conventional melanoma, we sought to evaluate their prognostic significance in spitzoid neoplasms. We analyzed tumors labeled as atypical Spitz tumor or spitzoid melanoma from 56 patients with available follow-up data for the association of TERT-p mutations, biallelic CDKN2A deletion, biallelic PTEN deletion, kinase fusions, BRAFINRAS mutations, nodal status, and histopathological parameters with risk of hematogenous metastasis. Four patients died of disseminated disease and 52 patients were alive and disease free without extranodal metastasis (median follow-up, 32.5 months). We found TERT-p mutations in samples from the 4 patients who developed hematogenous metastasis but in none of tumors from patients who had favorable outcomes. Presence of TERT-p mutations was the most significant predictor of haematogenous dissemination $(P<0.0001)$ among variables analyzed. We conclude that TERT-p mutations identify a clinically high-risk subset of patients with spitzoid tumors. Application of TERT-p mutational assays for risk stratification in the clinic requires large-scale validation.

Spitzoid neoplasms are melanocytic tumors with distinct histologic characteristics that more commonly develop during the first 2 decades of life. Since their initial description by Sophie Spitz in $1948^{1}$, the histologic diagnosis and appropriate management of spitzoid tumors have been controversial ${ }^{2-8}$.

Tumors with spitzoid morphology can present with a wide spectrum of biological properties, encompassing neoplasms that are entirely benign, called Spitz nevus, those with a low-grade or borderline malignant potential, termed atypical Spitz tumor (AST), and fully malignant neoplasms called spitzoid melanoma (SM). ASTs are tumors of intermediate malignancy that commonly spread to regional lymph nodes but do not progress to hematogenous metastasis ${ }^{9,10}$. On histological grounds, the distinction between an AST (tumors with metastatic capacity limited to regional lymph nodes) from SM (tumors with potential for extranodal metastasis) can be diagnostically challenging ${ }^{3}$. Sometimes lesions initially

${ }^{1}$ Departments of Pathology, St. Jude Children's Research Hospital, Memphis, Tennessee 38105, USA. ²Département de BioPathologie, Institut Curie, 26 rue d'Ulm, 75248, Paris cedex 05, France. ${ }^{3}$ Department of Dermatology, University Hospital Zurich, Gloriastrasse 31, CH-8091 Zurich, Switzerland. 'Departments of Biostatistics, St. Jude Children's Research Hospital, Memphis, Tennessee 38105, USA. 5Departments of Oncology, St. Jude Children's Research Hospital, Memphis, Tennessee 38105 , USA. Correspondence and requests for materials should be addressed to A.B. (email: armita.bahrami@stjude.org) 
diagnosed as AST are reclassified as melanoma once distant metastasis develops. To date, no single histopathological criterion ${ }^{7}$ or molecular marker is known to predict with certainty the risk of subsequent aggressive disease with these tumors.

Telomerase activity is crucial for tumorigenesis and cancer progression ${ }^{11,12}$. The activity of telomerase, the enzyme responsible for maintaining telomeric DNA during replication, is regulated by the telomerase reverse transcriptase (TERT) gen $\mathrm{e}^{13}$. Next-generation sequencing studies have identified somatic mutations in the core promoter region of TERT that by generating Ets/TCF transcription binding motifs increase the transcriptional activity of the gene ${ }^{14,15}$. TERT promoter (TERT-p) mutations have been found in $22 \%-71 \%$ of cutaneous melanoma in adult series ${ }^{14-19}$ and in the majority of conventional pediatric melanoma in a study from our group ${ }^{20}$, suggesting that they contribute to TERT regulation in melanoma. Interestingly, in our original series of pediatric melanoma, we found a hot-spot TERT-p mutation in the single patient with SM who died of disease but in no other patients with spitzoid tumors who had favorable outcomes ${ }^{20}$. We postulated that the molecular mechanism for maintaining telomere may be similar in SM and conventional melanoma. Herein, we investigated the presence of TERT-p mutations in 56 patients with histopathologically well-characterized atypical spitzoid neoplasms for whom follow-up information was available.

\section{Results}

Clinical Features. The clinical characteristics of patients are provided in Table S1. Tumors occurred in 33 female and 23 male patients aged 2-61 years (median, 9; mean, 14.6). They arose in skin of the lower extremity $(n=26)$, upper extremity $(n=9)$, face $(n=7)$, trunk $(n=6)$, ear $(n=5)$, and scalp $(n=3)$. Of the 42 patients who underwent sentinel lymph node evaluation, 21 (50\%) had at least 1 positive lymph node, of which 9 had extensive nodal metastasis. Fifty-two patients were alive with no evidence of disease at last follow-up (mean, 32.5 months). Four patients developed hematogenous metastasis and died of widespread disease.

Histologic Features. The histologic features of the 56 tumors are provided in Table S2. The Breslow tumor thickness ranged from 0.3 to $13.3 \mathrm{~mm}$ (median, $2.85 \mathrm{~mm}$ ) in the 52 tumors with a favorable behavior and 1.3 to $8 \mathrm{~mm}$ (median, $5.25 \mathrm{~mm}$ ) in the 4 tumors with an unfavorable behavior. The lesional diameter ranged from 1.5 to $17 \mathrm{~mm}$ (median, $6.5 \mathrm{~mm}$ ) in tumors with a favorable behavior and 4 to $12 \mathrm{~mm}$ (median, $11 \mathrm{~mm}$ ) for those with an unfavorable behavior. Ulceration was present in 10 of 52 (19\%) tumors with a favorable and in 3 of $4(75 \%)$ tumors with an unfavorable behavior. A high mitotic rate $\left(>5 / \mathrm{mm}^{2}\right)$ was seen in 5 of $52(10 \%)$ tumors with a favorable behavior and in 3 of $4(75 \%)$ tumors with an unfavorable behavior.

TERT Promoter Mutations. Samples from 4 of 56 patients contained 1 of the known hot-spot single nucleotide variations (SNVs), including 3 SNV 228 G > A (C > T) (Chr5:1295228/hg19), at position $-124 \mathrm{bp}$, and 1 tandem mutation 242/243 GG $>$ AA (CC > TT) (Chr5:1295242-1295243), at positions $-138 /-139$ bp from the ATG start site. The paired primary and metastatic tumor samples had similar variant mutations. All 4 patients with tumors harboring TERT-p mutations died from disseminated disease. In contrast, tumors in none of the 52 patients with a favorable clinical course carried these mutations (Fig. 1).

Kinase Fusions. A panel of break-apart fluorescence in situ hybridization (FISH) for ROS1, NTRK1, $A L K, B R A F$, and RET was successfully performed in 51 tumors. Gene rearrangement was found in 23 of $51(45 \%)$ tumors in mutually exclusive groups: ALK in $6(12 \%), R O S 1$ in $6(12 \%)$, NTRK1 in 5 (10\%), $B R A F$ in $4(8 \%)$, and $R E T$ in $2(4 \%)$. One of 4 tumors from patients with an unfavorable clinical course carried a $B R A F$ fusion (Fig. 1).

BRAF and NRAS Mutations. Of the 56 tumors, 3 (5\%) carried a BRAF mutation (2 V600 E in patients with a favorable and $1 \mathrm{~V} 600 \mathrm{~K}$ in a patient with an unfavorable clinical course). None of the tumors had an NRAS mutation (Fig. 1).

$C D K N_{2} A$ (p16). FISH identified biallelic CDKN2A deletion in 12 of 49 (24\%) successfully tested samples from patients with a favorable clinical course and in 2 of 4 (50\%) samples from patients with an unfavorable clinical course (Fig. 1). In some patients, the status of p16 was different between their primary and metastatic samples (see Discussion). As expected, biallelic CDKN2A deletion was predictive of loss of p16 by immunohistochemistry (Table S3). In 2 samples, immunohistochemical analysis showed loss of p16 expression without evidence of biallelic gene deletion by FISH (Table S3), suggesting that CDKN2A might be inactivated by mechanisms other than large deletion.

PTEN. Biallelic deletion of PTEN was found in 4 of 40 (10\%) samples from patients with a favorable clinical course and in none of the 3 successfully tested samples from patients with an unfavorable clinical course (Table 1). 


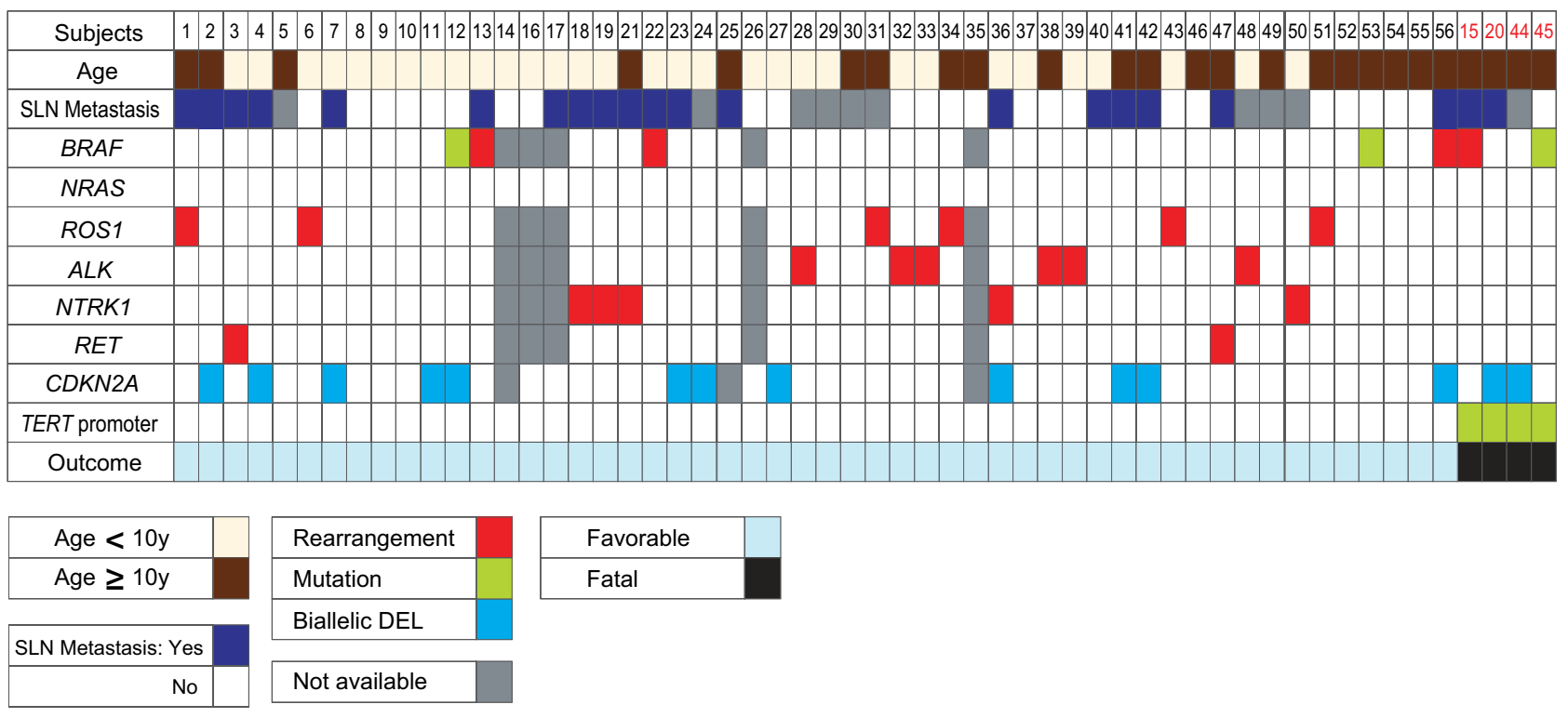

Figure 1. Association of kinase fusions, BRAF and NRAS mutations, biallelic CDKN2A deletion, and TERT promoter mutations with outcome in $\mathbf{5 6}$ patients with atypical spitzoid melanocytic neoplasm. Subject numbers in black font had a favorable clinical course and subject numbers in red font died of disseminated disease. Abbreviations: SLN, sentinel lymph node; DEL, deletion

Association Analysis. The presence of TERT-p mutations was significantly associated with the risk of extranodal metastatic disease or death $(P<0.0001)$ (Table 1$)$. In addition, age $\geq 10$ years, mitotic rate $>5 / \mathrm{mm}^{2}$, and ulceration were each associated with the risk of extranodal metastasis or death $(P<0.05)$. In contrast, no statistically significant association was found between the presence of biallelic loss of CDKN2A and extranodal metastasis or death $(P=0.56)$ (Table 1$)$. Gender, nodal metastasis, primary tumor thickness, and tumor infiltrating lymphocytes were also not associated with extranodal metastasis. Lesional diameter was marginally associated with extranodal metastasis $(P=0.054)$. The presence of TERT-p mutations was correlated with age $\geq 10$ years at diagnosis $(P=0.034)$, as well as mitotic rate $>5 / \mathrm{mm}^{2}$ and ulceration. A multiple-regression analysis to adjust the age effect was not feasible due to the small number of events (metastasis/death) in the cohort.

\section{Discussion}

ASTs account for the majority of so-called melanomas encountered in children. Patients with atypical spitzoid neoplasms have frequent sentinel lymph node involvement, but their outcomes are much better than for patients with similar-staged conventional melanoma ${ }^{21,22}$. Lallas et al. conducted a systemic review of the literature and found that having positive sentinel lymph nodes did not predict a worse outcome in patients with AST $^{9}$. In our study, 21 of $42(50 \%)$ patients who underwent sentinel nodal sampling had positive nodes and 2 (5\%) developed extranodal disease. Consistent with previous studies ${ }^{9,22-26}$, we find no correlation between regional nodal metastasis and subsequent development of aggressive disease in our patients $(P=0.61)$.

The difficulty in predicting the risk of hematogenous metastasis in patients with atypical spitzoid lesions has prompted extensive investigations. Sptaz et al. proposed a histopathologic grading scheme for risk stratification of pediatric AST ${ }^{27}$. We found a similar set of variables (age $\geq 10$ years, ulceration, and mitotic activity $>5 / \mathrm{mm}^{2}$ ) associated with a later development of extranodal metastasis, although no single factor by itself was predictive of outcomes. Similar to the study by Heidenreich $\mathrm{et} \mathrm{al.}{ }^{19}$, the presence of TERT-p mutations in our cohort correlated with histopathologic parameters of poor prognosis, such as mitotic activity and ulceration. It is known that spitzoid tumors developing in the first decade of life (prepubertal ages), irrespective of having histologic attributes of malignancy, do not progress to distant metastasis, except in the rarest instances ${ }^{21,28}$. The absence of TERT-p mutations in tumors from younger patients $(<10$ years of age) in our study is consistent with this observation, although the underlying biological mechanism for the phenomenon remains to be elucidated.

Activated kinase signaling pathways via chromosomal translocations are responsible for tumorigenesis in spitzoid melanocytic tumors ${ }^{29,30}$. Wiesner et al. found kinase fusions of NTRK1, ROS1, ALK, BRAF, or RET in $55 \%$ of Spitz nevi, $56 \%$ of ASTs, and $39 \%$ of SMs ${ }^{30}$. Similarly, kinase fusions were found across the entire biological spectrum of spitzoid neoplasms in our cohort, indicating that they cannot be applied as a means to predict the biological behavior of spitzoid lesions. 


\begin{tabular}{|c|c|c|}
\hline Variables & $\begin{array}{c}\text { Favorable } \\
\text { Outcome } \\
(n=52)\end{array}$ & $\begin{array}{c}\text { Unfavorable } \\
\text { Outcome }(n=4)\end{array}$ \\
\hline \multicolumn{3}{|c|}{ Nodal metastasis at diagnosis $(P=0.61)^{*}$} \\
\hline Absent & $20(51 \%)$ & $1(33 \%)$ \\
\hline Positive & $19(49 \%)$ & $2(67 \%)$ \\
\hline NA & 13 & 1 \\
\hline \multicolumn{3}{|c|}{ Biallelic CDKN2A deletion $(P=0.56)$} \\
\hline Yes & $12(24 \%)$ & $2(50 \%)$ \\
\hline No & $37(76 \%)$ & $2(50 \%)$ \\
\hline $\mathrm{NA}$ & 3 & 0 \\
\hline \multicolumn{3}{|c|}{ Biallelic PTEN deletion $(P=1.00)$} \\
\hline Yes & $4(10 \%)$ & $0(0 \%)$ \\
\hline No & $36(90 \%)$ & $3(100 \%)$ \\
\hline NA & 12 & 1 \\
\hline \multicolumn{3}{|c|}{ TERT-p mutation $(P<0.0001)$} \\
\hline Yes & $0(0 \%)$ & $4(100 \%)$ \\
\hline No & $52(100 \%)$ & $0(0 \%)$ \\
\hline \multicolumn{3}{|l|}{ Oncogene $(P=0.62)$} \\
\hline \multicolumn{3}{|l|}{ Kinase fusion } \\
\hline$A L K$ & $6(13 \%)$ & $0(0 \%)$ \\
\hline ROS1 & $6(13 \%)$ & $0(0 \%)$ \\
\hline NTRK1 & $5(11 \%)$ & $0(0 \%)$ \\
\hline RET & $2(4 \%)$ & $0(0 \%)$ \\
\hline$B R A F$ & $3(6 \%)$ & $1(25 \%)$ \\
\hline \multicolumn{3}{|l|}{$B R A F$ mutation } \\
\hline $\mathrm{V} 600 \mathrm{E}$ & $2(4 \%)$ & $0(0 \%)$ \\
\hline V600 K & $0(0 \%)$ & $1(25 \%)$ \\
\hline NRAS mutation & $0(0 \%)$ & $0(0 \%)$ \\
\hline Not found & $23(49 \%)$ & $2(50 \%)$ \\
\hline NA & 5 & 0 \\
\hline
\end{tabular}

Table 1. Association of molecular markers and nodal status at diagnosis with outcome in 56 patients with atypical spitzoid melanocytic neoplasms ( 52 with favorable and 4 with unfavorable clinical outcome). ${ }^{*} P$-values for association between each factor and risk of hematogenous metastasis/death

The discovery of recurrent DNA copy number gains and losses in melanoma, and the absence of such changes in nevi, prompted the development of diagnostic assays to assist proper classification of histologically challenging melanocytic lesions ${ }^{31,32}$. The multiprobe melanoma FISH assay targets the common regions of alterations in melanoma: 6p25 (targeting RREB1), 6q23 (MYB), 11q13 (CCND1), and 9p21 $(C D K N 2 A)^{33-36}$; whereas comparative genomic hybridization $(\mathrm{CGH})$ studies provide a genome wide view of copy number changes ${ }^{37,38}$. Although both assays are valuable tools to discriminate nevi from borderline or malignant lesions, their ability in risk stratification for spitzoid tumors is uncertain. In our series, samples from 23 patients showed multiple copy number changes by CGH studies or an abnormal result on multicolor melanoma FISH assay, which supported an SM diagnosis, but only in 1 patient a clinically malignant phenotype became apparent (Table S3).

Biallelic 9p21 deletion is proposed as a marker for spitzoid tumors that are at high risk for aggressive behavior. In a study by Gerami et al., of 37 pediatric patients with AST, 9 developed extensive locoregional disease and 2 developed distant metastasis. Tumors in 2 patients with distant metastasis and 7 of 9 patients with advanced locoregional disease harbored homozygous loss of 9p21, suggesting that this is a marker for disease progression ${ }^{39}$. We too observed the acquisition of biallelic deletion of CDKN $2 A$ (a reflection of tumor progression) in the nodal metastasis of at least 2 patients in whom the primary tumors retained both copies of the gene (Table S3), but neither patient developed extranodal metastasis in follow-up. As a whole in our series, the presence of biallelic CDKN2A deletion did not have a statistically significant association with the future risk of extranodal metastasis. Of the 4 patients who 
developed disseminated disease, 2 did not have biallellic CDKN2A loss in their primary or metastatic tumor and 2 had biallelic deletion in a subset of melanocytes that were not present at distant metastatic sites. Moreover, biallelic CDKN2A deletion was relatively common in the entire cohort, including in $23 \%$ of patients with a favorable clinical course. Overall, the findings from our study do not support that bialleclic 9p21 deletion is a reliable marker predictive of extranodal metastasis in spitzoid neoplasms.

Replicative immortality, a required element for overt malignant transformation in neoplastic cells, is commonly achieved by telomerase activation ${ }^{11-13,40}$. Several mechanisms are implicated in the control and reactivation of TERT in cancer cells, such as TERT gene copy gain or epigenetic modulation through TERT-p methylation ${ }^{41-43}$. In addition, mutations in the core promoter region of TERT have been shown to increase the transcriptional activity of telomerase and to be an independent marker of poor prognosis in various cancers, such as glioblastoma, thyroid carcinoma ${ }^{44-48}$, and cutaneous conventional melanoma ${ }^{16,17}$. The finding of TERT-p mutations in tumors from patients with fatal outcome in our series, and its absence in those with favorable behavior, strongly suggest that TERT-p mutations are a predictive marker of aggressive clinical behavior in patients with spitzoid lesions.

The negative predictive value of TERT-p mutations, on the other hand, requires further validation. TERT-p mutations may be acquired late during multistep melanoma development ${ }^{49}$. Conceivably, subclones of immortalized melanocytes with TERT-p mutations might be present in a lesion but be missed if below the detection level of standard sequencing assays. Moreover, the diverse mechanisms by which cancer cells maintain telomere length suggest that alternative mechanisms may be enacted alone or with TERT-p mutations to restore telomere length in melanocytes ${ }^{41,42}$. In addition, the follow-up time in our cohort was not extended enough to document the ultimate long-term outcomes of patients with wild-type TERT-p. One of our patients presented with disseminated disease 10 years after an initial diagnosis of AST (see Supplementary material). To prove the reliability and reproducibility of TERT-p mutations as a screening tool in the clinic, the assay needs to be evaluated on primary-site spitzoid neoplasms in large-scale studies with long term follow-up. We are currently collaborating with the Pediatric Melanoma Registry, an international multiinstitutional registry of pediatric melanoma based at the University of Pittsburgh, to work toward this aim.

The unpredictable clinical course of patients with spitzoid neoplasms has made the optimal management of these patients debatable, often leading to overtreatment of patients at minimal risk for disease progression. The use of TERT-p mutations as a marker of aggressive disease can help stratify a subgroup of patients with spitzoid tumors that likely behave in a malignant fashion and for whom more intensive therapies are warranted.

\section{Materials and methods}

Tissue Specimens. The study was approved by the institutional review board at St. Jude Children's Research Hospital and the methods were carried in accordance with the approved guidelines. Written informed consent was not required under a HIPAA waiver IRB approval.

Tissue specimens from patients diagnosed with AST or SM were obtained from the surgical pathology archives. Inclusion criteria were as follows: (1) lesions showing some histologic features of Spitz nevus and meeting previously described criteria for AST and SM ${ }^{4,20,21,50}$; (2) availability of sufficient tissue for sequencing assays; and (3) availability of demographic and follow-up information. A comprehensive histologic evaluation by study investigators (RLB, RD, and $A B$ ) identified specimens from 56 patients with atypical spitzoid neoplasms (labeled as SM in 33 and AST in 23), including specimens from 5 previously reported patients ${ }^{20}$. The study material included formalin-fixed paraffin-embedded (FFPE) tissue specimens consisting of primary tumors $(n=49)$, paired primary and metastatic tumors $(n=5)$, and metastatic tumors $(n=2)$ from 56 patients.

Histopathologic Parameters. The following histopathologic parameters were considered in the statistical analysis: (1) primary tumor Breslow thickness (T1 to T4); (2) horizontal lesional diameter (1-5 mm; 6-10 mm; >10 mm); (3) ulceration (present; absent); (4) mitotic rate ( $<1$ per $\mathrm{mm}^{2} ; 1-5$ per $\mathrm{mm}^{2} ; 6-10 \mathrm{~mm}^{2}$; >10 $\mathrm{mm}^{2}$ ); (5) tumor-infiltrating lymphocytes (absent; brisk; non-brisk); (6) regional lymph node metastasis (absent; small deposits; large deposits); (7) biallelic CDKN2A deletion (yes; no); (8) biallelic PTEN deletion (yes; no); and (8) TERT-p mutations (yes; no).

Mutational Analysis of BRAF, NRAS, and TERT Promoter. FFPE tumor sections were manually microdissected guided by H\&E slides to obtain at least $50 \%$ tumor purity in the material used for DNA extraction. Genomic DNA was extracted according to the manufacturer's protocol using Maxwell ${ }^{\circledR}$ 16 FFPE Plus LEV DNA Purification Kit (Promega). Mutational hotspots for BRAF (exon 15), NRAS (exons 1 and 2), and a portion of TERT-p (HG19 coordinates, chr5: 1295151-1295347) were screened in genomic tumor DNA of the 56 tumors. PCRs were performed, using GoTaq ${ }^{\circledR}$ Long PCR Master Mix (Promega, Madison, WI) or AmpliTaq Gold ${ }^{\circledR} 360$ Master Mix (Applied Biosystems, Foster City, CA) using amplification primers as previously described ${ }^{20}$. Direct sequencing of PCR products was performed using BigDye version 3.1 and a 3730XL DNA analyzer (Applied Biosystems, Foster City, CA). Results were screened using CLC Main Workbench sequence analysis software version 6.0.2 (CLC bio, Cambridge, MA). 
Fluorescence in situ Hybridization. BAC clones (BACPAC Resources, Oakland, CA) were used to develop copy number and break-apart probes for the following genes: CDKN2A (RP11-149I2) + 9q control (RP11-235C23), PTEN (RP11-2553L21) + 10p control (RP11-254A5 \& RP11-322I2), BRAF (RP11-837G3 \& RP11-948O19), NTRK1 (CH17-67O18 \& RP11-1038N13), RET (RP11-124O11 \& RP11718J13), ROS1 (RP11-103F10 \& RP11-1059G13), and ALK (CytoCell, Cat\# LPS 019-A, Cambridge, UK). Dual-color FISH was applied on $4-\mu \mathrm{m}$ FFPE sections as previously described ${ }^{20}$. FISH was successfully performed for a panel of kinase fusions (51 samples), copy number CDKN2A (58 primary/metastatic samples), and copy number PTEN (43 samples).

Immunohistochemical Analysis. FFPE tumor sections were processed for immunohistochemical analysis for p16 (JC8; Santa Cruz) as previously described ${ }^{20}$.

Statistical Analysis. Contingency tables were generated to study associations between outcome and risk factors. Pearson's chi-square exact tests were used to test the associations.

\section{References}

1. Spitz, S. Melanomas of childhood. The American journal of pathology 24, 591-609 (1948).

2. Braun, R. P. et al. Agreement of dermatopathologists in the evaluation of clinically difficult melanocytic lesions: how golden is the 'gold standard'? Dermatology 224, 51-58, doi:10.1159/000336886 (2012).

3. Barnhill, R. L. et al. Atypical Spitz nevi/tumors: lack of consensus for diagnosis, discrimination from melanoma, and prediction of outcome. Human pathology 30, 513-520 (1999).

4. Spatz, A. \& Barnhill, R. L. The Spitz tumor 50 years later: revisiting a landmark contribution and unresolved controversy. Journal of the American Academy of Dermatology 40, 223-228 (1999).

5. Reed, D., Kudchadkar, R., Zager, J. S., Sondak, V. K. \& Messina, J. L. Controversies in the evaluation and management of atypical melanocytic proliferations in children, adolescents, and young adults. Journal of the National Comprehensive Cancer Network: JNCCN 11, 679-686 (2013).

6. Cerroni, L. et al. Melanocytic tumors of uncertain malignant potential: results of a tutorial held at the XXIX Symposium of the International Society of Dermatopathology in Graz, October 2008. The American journal of surgical pathology 34, 314-326, doi:10.1097/PAS.0b013e3181cf7fa0 (2010)

7. Gerami, P. et al. Histomorphologic assessment and interobserver diagnostic reproducibility of atypical spitzoid melanocytic neoplasms with long-term follow-up. The American journal of surgical pathology 38, 934-940, doi:10.1097/PAS.0000000000000198 (2014).

8. Mones, J. M. \& Ackerman, A. B. “Atypical” Spitz's nevus, "malignant" Spitz's nevus, and "metastasizing” Spitz's nevus: a critique in historical perspective of three concepts flawed fatally. The American Journal of dermatopathology 26, 310-333 (2004).

9. Lallas, A. et al. Atypical Spitz tumours and sentinel lymph node biopsy: a systematic review. The lancet oncology 15, e178-183, doi:10.1016/S1470-2045(13)70608-9 (2014).

10. Berk, D. R., LaBuz, E., Dadras, S. S., Johnson, D. L. \& Swetter, S. M. Melanoma and melanocytic tumors of uncertain malignant potential in children, adolescents and young adults-the Stanford experience 1995-2008. Pediatr Dermatol 27, 244-254, doi:10.1111/j.1525-1470.2009.01078.x (2010).

11. Stewart, S. A. \& Weinberg, R. A. Telomerase and human tumorigenesis. Seminars in cancer biology 10, 399-406, doi:10.1006/ scbi.2000.0339 (2000)

12. Hanahan, D. \& Weinberg, R. A. Hallmarks of cancer: the next generation. Cell 144, 646-674, doi:10.1016/j.cell.2011.02.013 (2011).

13. Hahn, W. C. et al. Creation of human tumour cells with defined genetic elements. Nature 400, 464-468, doi:10.1038/22780 (1999).

14. Horn, S. et al. TERT promoter mutations in familial and sporadic melanoma. Science 339, 959-961, doi:10.1126/science.1230062 (2013).

15. Huang, F. W. et al. Highly recurrent TERT promoter mutations in human melanoma. Science 339, 957-959, doi:10.1126/ science.1229259 (2013).

16. Populo, H., Lopes, J. M., Sobrinho-Simoes, M. \& Soares, P. RE: TERT promoter mutation status as an independent prognostic factor in cutaneous melanoma. Journal of the National Cancer Institute 107, doi:10.1093/jnci/djv049 (2015).

17. Griewank, K. G. et al. TERT promoter mutation status as an independent prognostic factor in cutaneous melanoma. Journal of the National Cancer Institute 106, doi:10.1093/jnci/dju246 (2014).

18. Populo, H. et al. TERT promoter mutations in skin cancer: the effects of sun exposure and X-irradiation. The Journal of investigative dermatology 134, 2251-2257, doi:10.1038/jid.2014.163 (2014).

19. Heidenreich, B. et al. Telomerase reverse transcriptase promoter mutations in primary cutaneous melanoma. Nature communications 5, 3401, doi:10.1038/ncomms4401 (2014).

20. Lu, C. et al. The Genomic Landscape of Childhood and Adolescent Melanoma. The Journal of investigative dermatology, doi:10.1038/jid.2014.425 (2014).

21. Paradela, S. et al. Spitzoid melanoma in children: clinicopathological study and application of immunohistochemistry as an adjunct diagnostic tool. Journal of cutaneous pathology 36, 740-752, doi:10.1111/j.1600-0560.2008.01153.x (2009).

22. Ludgate, M. W. et al. The atypical Spitz tumor of uncertain biologic potential: a series of 67 patients from a single institution. Cancer 115, 631-641, doi:10.1002/cncr.24047 (2009).

23. Lohmann, C. M., Coit, D. G., Brady, M. S., Berwick, M. \& Busam, K. J. Sentinel lymph node biopsy in patients with diagnostically controversial spitzoid melanocytic tumors. The American journal of surgical pathology 26, 47-55 (2002).

24. Su, L. D., Fullen, D. R., Sondak, V. K., Johnson, T. M. \& Lowe, L. Sentinel lymph node biopsy for patients with problematic spitzoid melanocytic lesions: a report on 18 patients. Cancer 97, 499-507, doi:10.1002/cncr.11074 (2003).

25. Busam, K. J. \& Pulitzer, M. Sentinel lymph node biopsy for patients with diagnostically controversial Spitzoid melanocytic tumors? Advances in anatomic pathology 15, 253-262, doi:10.1097/PAP.0b013e31818323ac (2008).

26. Hung, T. et al. Sentinel lymph node metastasis is not predictive of poor outcome in patients with problematic spitzoid melanocytic tumors. Human pathology 44, 87-94, doi:10.1016/j.humpath.2012.04.019 (2013).

27. Spatz, A., Calonje, E., Handfield-Jones, S. \& Barnhill, R. L. Spitz tumors in children: a grading system for risk stratification. Archives of dermatology 135, 282-285 (1999).

28. Averbook, B. J. et al. Pediatric melanoma: analysis of an international registry. Cancer 119, 4012-4019, doi:10.1002/cncr.28289 (2013). 
29. Botton, T. et al. Recurrent BRAF kinase fusions in melanocytic tumors offer an opportunity for targeted therapy. Pigment cell \& melanoma research 26, 845-851, doi:10.1111/pcmr.12148 (2013).

30. Wiesner, T. et al. Kinase fusions are frequent in Spitz tumours and spitzoid melanomas. Nature communications 5, 3116, doi:10.1038/ncomms4116 (2014).

31. Bastian, B. C., LeBoit, P. E., Hamm, H., Brocker, E. B. \& Pinkel, D. Chromosomal gains and losses in primary cutaneous melanomas detected by comparative genomic hybridization. Cancer research 58, 2170-2175 (1998).

32. Bastian, B. C., Olshen, A. B., LeBoit, P. E. \& Pinkel, D. Classifying melanocytic tumors based on DNA copy number changes. American Journal of Pathology 163, 1765-1770, doi:10.1016/S0002-9440(10)63536-5 (2003).

33. Gerami, P. et al. Fluorescence in situ hybridization (FISH) as an ancillary diagnostic tool in the diagnosis of melanoma. The American journal of surgical pathology 33, 1146-1156, doi:10.1097/PAS.0b013e3181a1ef36 (2009).

34. Gammon, B., Beilfuss, B., Guitart, J. \& Gerami, P. Enhanced detection of spitzoid melanomas using fluorescence in situ hybridization with $9 \mathrm{p} 21$ as an adjunctive probe. The American journal of surgical pathology 36, 81-88, doi:10.1097/ PAS.0b013e31822d5ff8 (2012).

35. Kerl, K. et al. A proposal for improving multicolor FISH sensitivity in the diagnosis of malignant melanoma using new combined criteria. The American Journal of dermatopathology 34, 580-585, doi:10.1097/DAD.0b013e3182433f3a (2012).

36. Gerami, P. et al. A highly specific and discriminatory FISH assay for distinguishing between benign and malignant melanocytic neoplasms. The American journal of surgical pathology 36, 808-817, doi:10.1097/PAS.0b013e31824blefd (2012).

37. Bastian, B. C., Wesselmann, U., Pinkel, D. \& Leboit, P. E. Molecular cytogenetic analysis of Spitz nevi shows clear differences to melanoma. The Journal of investigative dermatology 113, 1065-1069, doi:10.1046/j.1523-1747.1999.00787.x (1999).

38. Harvell, J. D. et al. High-resolution array-based comparative genomic hybridization for distinguishing paraffin-embedded Spitz nevi and melanomas. Diagnostic molecular pathology : the American journal of surgical pathology, part B 13, 22-25 (2004).

39. Gerami, P. et al. Outcomes of Atypical Spitz Tumors With Chromosomal Copy Number Aberrations and Conventional Melanomas in Children. The American journal of surgical pathology, doi:10.1097/PAS.0b013e31828fc283 (2013).

40. Counter, C. M. et al. Telomerase activity is restored in human cells by ectopic expression of hTERT (hEST2), the catalytic subunit of telomerase. Oncogene 16, 1217-1222, doi:10.1038/sj.onc.1201882 (1998).

41. Daniel, M., Peek, G. W. \& Tollefsbol, T. O. Regulation of the human catalytic subunit of telomerase (hTERT). Gene 498, 135-146, doi:10.1016/j.gene.2012.01.095 (2012).

42. Puig-Butille, J. A. et al. Genetic alterations in RAS-regulated pathway in acral lentiginous melanoma. Experimental dermatology 22, 148-150, doi:10.1111/exd.12080 (2013).

43. Castelo-Branco, P. et al. Methylation of the TERT promoter and risk stratification of childhood brain tumours: an integrative genomic and molecular study. The lancet oncology 14, 534-542, doi:10.1016/s1470-2045(13)70110-4 (2013).

44. Simon, M. et al. TERT promoter mutations: a novel independent prognostic factor in primary glioblastomas. Neuro-oncology 17, 45-52, doi:10.1093/neuonc/nou158 (2015).

45. Huse, J. T. TERT promoter mutation designates biologically aggressive primary glioblastoma. Neuro-oncology 17, 5-6, doi:10.1093/ neuonc/nou318 (2015)

46. Vinagre, J. et al. Telomerase promoter mutations in cancer: an emerging molecular biomarker? Virchows Archiv : an international journal of pathology 465, 119-133, doi:10.1007/s00428-014-1608-4 (2014).

47. Shi, X. et al. Association of TERT Promoter Mutation 1,295,228 C > T with BRAF V600E Mutation, Older Patient Age, and Distant Metastasis in Anaplastic Thyroid Cancer. The Journal of clinical endocrinology and metabolism, jc20143606, doi:10.1210/ jc.2014-3606 (2015).

48. Gandolfi, G. et al. TERT promoter mutations are associated with distant metastases in Papillary Thyroid Carcinoma. European journal of endocrinology / European Federation of Endocrine Societies, doi:10.1530/EJE-14-0837 (2015).

49. Soo, J. K. et al. Malignancy without immortality? Cellular immortalization as a possible late event in melanoma progression. Pigment cell \& melanoma research 24, 490-503, doi:10.1111/j.1755-148X.2011.00850.x (2011).

50. Barnhill, R. L., Flotte, T. J., Fleischli, M. \& Perez-Atayde, A. Cutaneous melanoma and atypical Spitz tumors in childhood. Cancer 76, 1833-1845 (1995).

\section{Acknowledgement}

Research reported in this publication was supported in part by the National Cancer Institute of the National Institutes of Health under Award Number P30CA021765 and by the American Lebanese Syrian Associated Charities (ALSAC). This work was presented in part at the American Society of Clinical Oncology Annual Meeting, Chicago, June 2014. The authors would like to thank Drs. John Kirkwood and Vani Shanker for their critical review of the manuscript, and Valerie McPherson and Dana Hawkins for technical and administrative support.

\section{Author Contributions}

Manuscript writing: All authors Collection and assembly of data: S.L., R.L.B., R.D., J.D., A.P. and A.B. Data analysis and interpretations: S.L., J.D., J.W., A.P. and A.B. Final approval of manuscript: All authors.

\section{Additional Information \\ Supplementary information accompanies this paper at http://www.nature.com/srep \\ Competing financial interests: The authors declare no competing financial interests.}

How to cite this article: Lee, S. et al. TERT Promoter Mutations Are Predictive of Aggressive Clinical Behavior in Patients with Spitzoid Melanocytic Neoplasms. Sci. Rep. 5, 11200; doi: 10.1038/srep11200 (2015).

This work is licensed under a Creative Commons Attribution 4.0 International License. The images or other third party material in this article are included in the article's Creative Commons license, unless indicated otherwise in the credit line; if the material is not included under the Creative Commons license, users will need to obtain permission from the license holder to reproduce the material. To view a copy of this license, visit http://creativecommons.org/licenses/ by/4.0/ 